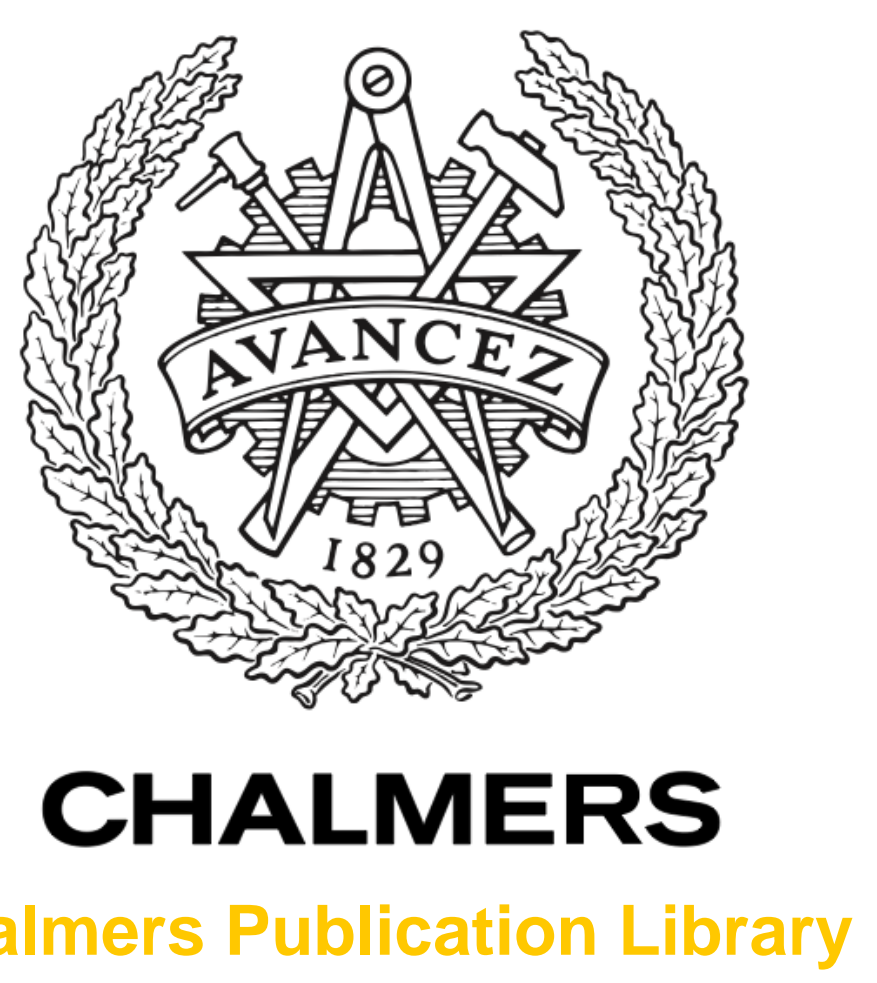

Chalmers Publication Library

Spectrally efficient four-dimensional modulation

This document has been downloaded from Chalmers Publication Library (CPL). It is the author's version of a work that was accepted for publication in:

2012 Optical Fiber Communication Conference and Exposition and the National Fiber Optic Engineers Conference, OFC/NFOEC 2012, Los Angeles, CA, 4 March through 8 March 2012

Citation for the published paper:

Karlsson, M. ; Agrell, E. (2012) "Spectrally efficient four-dimensional modulation". 2012 Optical Fiber Communication Conference and Exposition and the National Fiber Optic Engineers Conference, OFC/NFOEC 2012, Los Angeles, CA, 4 March through 8 March 2012(Article number 6192053), pp. OTu2C1.

http://dx.doi.org/10.1364/OFC.2012.OTu2C.1

Downloaded from: http://publications.lib.chalmers.se/publication/159708

Notice: Changes introduced as a result of publishing processes such as copy-editing and formatting may not be reflected in this document. For a definitive version of this work, please refer to the published source. Please note that access to the published version might require a subscription. 


\title{
Spectrally efficient four-dimensional modulation
}

\author{
Magnus Karlsson (1) and Erik Agrell (2) \\ (1) Department of Microtechnology and Nanoscience, (2) Department of Signals and Systems, \\ Chalmers University of Technology, SE-412 96 Göteborg, Sweden \\ email:magnus.karlsson@chalmers.se
}

\begin{abstract}
We quantify the performance of regular four-dimensional constellations with high spectral efficiency, and compare the sensitivity gain relative to rectangular QAM. Gains up to 1.51 and $1.97 \mathrm{~dB}$ over the corresponding QAM formats can be achieved.

OCIS codes: (060.4080) Modulation; (060.4510) Optical communications
\end{abstract}

\section{Introduction}

Increasing the spectral efficiency (SE) is one important approach to extending the performance of optical communication systems to deal with the increasing demands for capacity. This is usually done by increasing the number of signaling levels in rectangular quadrature amplitude modulation (QAM) systems, with recent examples being demonstrations of 64- [1] and 512-QAM [2]. However this always comes with an increased demand for signal power, which is ultimately limited by fiber nonlinearities. To increase the SE further while maintaining a reasonable signaling power, one needs to increase the constellation dimensionality, by coding and/or modulation. Since coherent optical systems have a four-dimensional (4d) constellation space [3,4] a natural first approach is to find suitable 4d constellations, e.g. optimized for best asymptotic sensitivity, and such constellations with up to 24 levels have been proposed [4-6].

For the optimized 8-point structure (often referred to as polarization switched quadrature phase shift keying, PS-QPSK [4]) there have been experimental realizations [7,8]. At higher spectral efficiencies, however, the optimal constellations require transmitter and receiver algorithms based on table lookup and full search, which implies a prohibitive implementation complexity. Therefore, as in the single-polarization case (two-dimensional inphase/quadrature modulation, I/Q), regular but suboptimal constellations are mainly considered for high spectral efficiency requirements. However, recently PS-QPSK was generalized to higher spectral efficiencies by Coelho and Hanik [9]. By using set portioning (SP) to realize subsets of regular 4d-QAM constellations they realized structured 32- and 128-level SP-QAM formats with good performance, yet reasonable complexity in generation and detection.

In this invited paper, we analyze the theoretical performance of these and other structured $4 \mathrm{~d}$ modulation formats for high spectral efficiencies. We compare them with numerically optimized constellations, interpret them geometrically, and explain the obtained gains analytically.

\section{System model and figures of merit}

For long-haul coherent optical links, the dominant impairment comes from amplified spontaneous emission in the optical amplifiers, and such links are well modeled by the additive white Gaussian noise channel. With this model, assuming a high signal-to-noise ratio and no forward error correction coding, the performance of an $N$-dimensional, $M$-level modulation format is well captured by two figures-of-merit, the spectral efficiency $S E=2 \log _{2}(M) / N$ and the (asymptotic) power efficiency $P E=d_{\min }^{2} \log _{2}(M) /\left(4 E_{\mathrm{s}}\right)$, where $E_{\mathrm{s}}$ is the average energy per symbol and $d_{\min }$ is the smallest Euclidean distance between any pair of symbols in the constellation. The spectral efficiency, in bits per symbol per polarization, equals the bitrate gain over QPSK at the same bandwidth, and the power efficiency $P E$ in $\mathrm{dB}$ equals the asymptotic sensitivity gain over QPSK at the same bitrate.

With the definitions above, binary phase-shift keying (BPSK), QPSK and dual polarization (DP)-QPSK all have $S E=2$ and $P E=1$. Furthermore, the spectral and power efficiencies of one-dimensional pulse amplitude modulation (PAM) are related by

$$
P E_{\mathrm{PAM}}=\frac{3 S E}{2\left(2^{S E}-1\right)}
$$

for any number of levels, and the same relation holds for any square QAM, DP-QAM or any higher $N$-dimensional generalization of PAM. Such constellations are said to be a subset of the cubic lattice, which is formed by all points with integer coordinates. Following Forney [10], we will in this paper use PAM as a baseline system, against which other modulation formats at the same spectral efficiency are compared. Generalizing, (1) will be used as the performance reference even at spectral efficiencies for which no PAM system exist. 

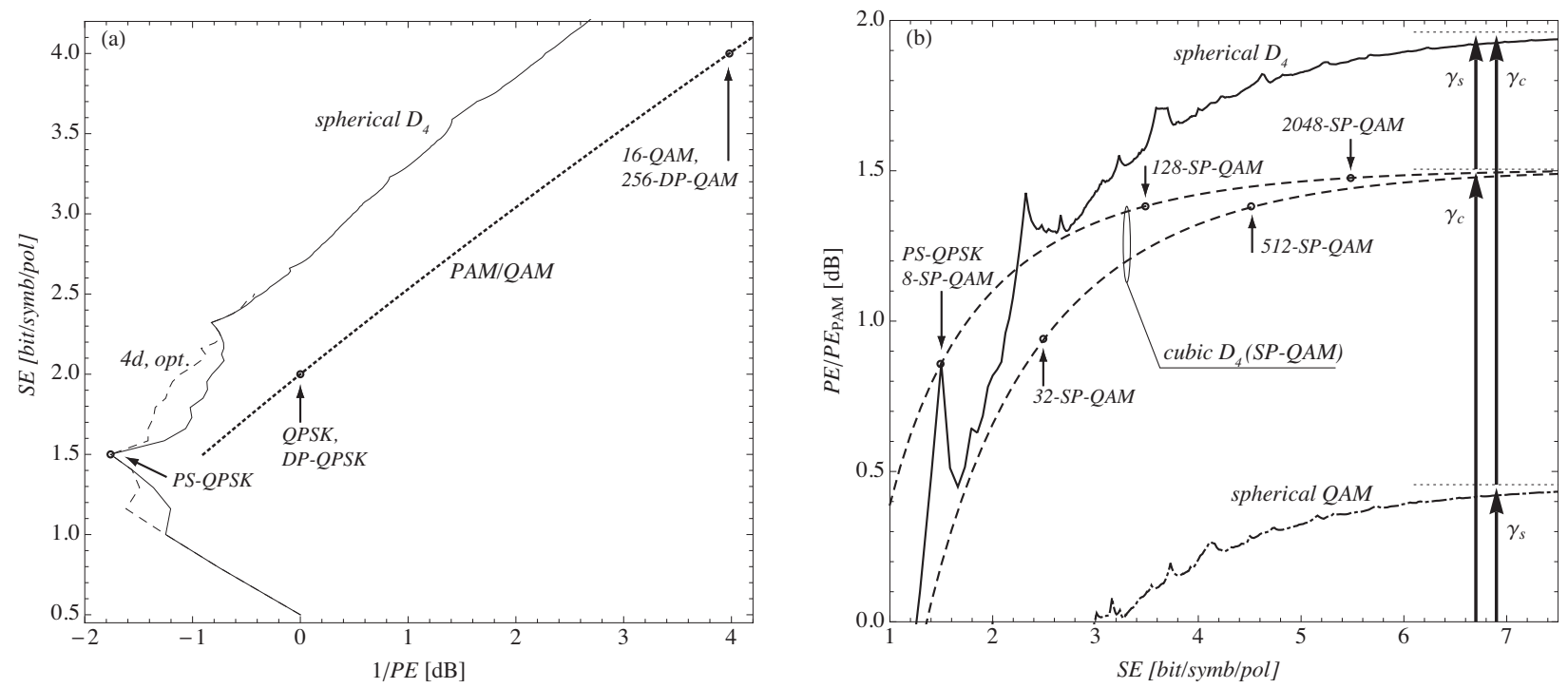

Fig. 1: (a) Performance of the best $D_{4}$-lattice-based constellations, (solid line, marked "spherical $D_{4}$ "), compared with optimized, unstructured, constellations (dashed, "4d, opt.") from [4], and the PAM/QAM formats from (1). (b) Power efficiency gain over PAM for spherical subsets of $D_{4}$ and QAM (solid lines) and SP-QAM (dashed lines, from (2)). Some specific formats are shown with dots and arrows, and the coding gain $\left(\gamma_{\mathrm{c}}=1.51 \mathrm{~dB}\right)$ and shaping gain $\left(\gamma_{\mathrm{s}}=0.46 \mathrm{~dB}\right)$ have been illustrated in $(\mathrm{b})$.

\section{Multilevel four-dimensional constellations}

In spectrally efficient modulation, i.e. for large $M$, it is well known that the optimum constellations approach regular sphere packing structures, so called lattices, shaped by a spherical boundary [10,11]. The regular structure is often broken near the boundary, but the influence of such irregularities diminishes as the number of points $M$ increases for a fixed dimension $N$. The optimal structures are the hexagonal lattice in two dimensions and the so-called $D_{4}$ lattice in four dimensions. ${ }^{1}$ The regular structure is good news for the system designer, since it can be exploited in known, low-complexity transmitter [12] and receiver [13] algorithms.

Guided by this principle, we design multilevel constellations in four dimensions by selecting $M$-ary spherical subsets of the $D_{4}$ lattice. The size of the sphere was chosen to contain the desired number $M$ of lattice points, and its center was adjusted to minimize $E_{\mathrm{s}}$ in each case. The power and spectral efficiencies of the obtained constellations are shown with the solid line in Fig. 1 (a), along with the performance of the optimized $M$-level constellations for $S E \leq 2.5$ from [4]. The baseline curve $P E_{\mathrm{PAM}}$ is also shown for reference together with some selected formats. As expected, the lattice-based constellations are weaker for small values of $M$, with the notable exception of $M=8$, because PS-QPSK is a subset of the $D_{4}$ lattice. On the other hand, there is practically nothing to gain from optimizing constellations at higher spectral efficiencies $S E \geq 2.2$. The multilevel lattice-based constellations perform 1.5-1.7 dB better than the baseline.

Although advantageous from a power efficiency viewpoint, a spherical boundary is complicated to implement in practice. A compromise between spherical $D_{4}$ constellations and DP-QAM can be designed by bounding the $D_{4}$ subconstellations by a $4 \mathrm{~d}$ cube rather than a sphere. Such structures was proposed in [9], and referred to as set partitioning (SP)-QAM. They can be conveniently constructed from DP-QAM by removing half of the points, i.e. by replacing the cubic with the $D_{4}$ lattice. Conversely, the union of two $2^{4 m}$-DP-QAM constellations, suitably shifted in relation to each other, forms a $2^{4 m+1}$-level SP-QAM constellation. Similarly, if every second point in a $2^{4 m}$-DP-QAM constellation is removed, a $2^{4 m-1}$-level SP-QAM constellation is obtained (e.g., PS-QPSK, or 8-SP-QAM, if $m=1$ ). These two constructions both generate the same structure (the $D_{4}$ lattice), although with different orientations relative to the bounding cube. Among the advantages of the SP-QAM constellations, in comparison with constellations with a spherical boundary, can be mentioned that their sizes are powers of two (assuming that the same holds for the constituent DP-QAM constellation), which makes the bit-to-symbol mappings trivial, and that very efficient decoding methods are available [13]. The drawback is the reduced power efficiency, relative to the spherically shaped constellation. A

\footnotetext{
${ }^{1}$ The $D_{4}$ lattice consist of all $4 \mathrm{~d}$ points with integer coordinates, where the coordinate sum is an even number. It can be seen as the $4 \mathrm{~d}$ generalization of a checkerboard pattern.
} 
Table 1: Comparison between different 4d QAM and SP-QAM formats.

\begin{tabular}{ccccc}
\hline Lattice & Format & No. of levels $M$ & $2 \cdot S E[\mathrm{bit} / \mathrm{s} / \mathrm{Hz}]$ & $P E[\mathrm{~dB}]$ \\
\hline \hline Cubic & DP-QPSK & 16 & 4 & 0 \\
Cubic & 256-DP-QAM & 256 & 8 & -3.98 \\
\hline$D_{4}$ & PS-QPSK & 8 & 3 & 1.76 \\
$D_{4}$ & 32-SP-QAM & 32 & 5 & 0 \\
$D_{4}$ & 128-SP-QAM & 128 & 7 & -1.55 \\
$D_{4}$ & 512-SP-QAM & 512 & 9 & -3.68 \\
\hline \hline
\end{tabular}

similar relation as (1) between $S E$ and $P E$ can be obtained also for SP-QAM as

$$
P E_{\mathrm{SP}-\mathrm{QAM}}=\frac{3 S E}{2^{S E+1 / 2}-1}, \text { if } S E+\frac{1}{2} \text { is even, } \quad P E_{\mathrm{SP}-\mathrm{QAM}}=\frac{3 S E}{2^{S E+1 / 2}-\frac{1}{2}}, \text { if } S E+\frac{1}{2} \text { is odd. }
$$

The power gain for spherical and cubically shaped $D_{4}$ structures over the baseline system are shown in Fig. 1 (b), with the SP-QAM relations (2) dashed and some specific $M$-ary SP-QAM formats marked. For completeness, we also include another hybrid between DP-QAM and the spherically shaped $D_{4}$ constellations, namely, spherically shaped QAM constellations. It can be seen that the power gain of spherical $D_{4}$ (and hence of optimal $4 \mathrm{~d}$ constellations) over PAM approaches $1.96 \mathrm{~dB}$ asymptotically, representing the horizontal gap in Fig. 1 (a). According to [10], this gain consists of two parts, which are independent of each other at high spectral efficiencies: a coding gain $\gamma_{\mathrm{c}}$, which comes from replacing the cubic lattice (as in regular QAM) with $D_{4}$, and a shape gain $\gamma_{\mathrm{s}}$, which comes from replacing the bounding $4 \mathrm{~d}$ cube with a $4 \mathrm{~d}$ sphere. The exact gains are $\gamma_{\mathrm{c}}=\sqrt{2}=1.51 \mathrm{~dB}$ and $\gamma_{\mathrm{s}}=\pi /(2 \sqrt{2})=0.46 \mathrm{~dB}$, resp., which are also shown inf Fig. 1 (b). (The corresponding gains in two dimensions are $\gamma_{c}=0.62 \mathrm{~dB}$ for the hexagonal lattice and a mere $\gamma_{\mathrm{s}}=0.20 \mathrm{~dB}$ for the circle, which explains why standard QAM is still so popular in single-polarization I/Q modulation.)

\section{Discussion and Conclusions}

Table 1 compares sensitivities and spectral efficiencies of the lowest-order DP-QAM and SP-QAM formats. It is interesting to note that 32-SP-QAM has the same sensitivity as DP-QPSK, while transmitting one more bit per symbol. In addition, 512-SP-QAM transmits 9 bits per symbol relative to 8 for dual-polarization 16-QAM (256-DP-QAM) with better sensitivity, and with only marginally increased demodulation complexity. However, new coherent decoding algorithms for phase- and polarization tracking may need to be developed, as discussed in [9].

To conclude, we have compared various design strategies for spectrally efficient four-dimensional modulation formats. The most reasonable tradeoff between complexity and performance is obtained by cubically shaped subsets of the $D_{4}$ lattice, also known as SP-QAM. At high spectral efficiencies, these formats capture $1.51 \mathrm{~dB}$ out of the maximum available $1.96 \mathrm{~dB}$ gain over the baseline QAM system.

We gratefully acknowledge funding from Vinnova within the IKT grant (no. 2007-02930) and the Swedish Strategic Research Foundation (SSF) (no. RE07-0026). We also acknowledge inspiring discussions with colleagues within the Chalmers FORCE center.

\section{References}

1. T. Kobayashi et al., OFC'11, Paper OThF6, 2011

2. S. Okamoto et al., ECOC'10, Paper PD 2.3, 2010

3. R. Cusani et al., J. Lightw. Technol., vol. 10, no. 6, pp. 777-786, 1992.

4. E. Agrell and M. Karlsson, J. Lightw. Technol., vol. 27, pp. 5115-5126, 2009.

5. H. Bülow, OFC'09, Paper OWG2, 2009.

6. M. Karlsson and E. Agrell, Ch. 5 in Impact of Nonlinearities on Fiber Optic Communication (ed. S. Kumar). Springer, 2011.
7. M. Sjödin et al., Opt. Exp., vol. 19, pp. 7839-7846, 2011.

8. L. Nelson et al., Opt. Exp., vol. 19, pp.10849-10856, 2011.

9. L. Coelho and N. Hanik, ECOC'11, Paper Mo.2.B.4, 2011

10. G. D. Forney, Jr. and L.-F. Wei, IEEE J. Sel. Ar. Comm., vol. 7, pp. 877-892, 1989.

11. J. H. Conway and N. J. A. Sloane, Sphere Packings, Lattices and Groups, 3rd ed., Springer, 1999.

12. J. H. Conway and N. J. A. Sloane, IEEE Trans. Inf. Theory, vol. 29, pp. 820-824, 1983.

13. J. H. Conway and N. J. A. Sloane, IEEE Trans. Inf. Theory, vol. 28, pp. 227-232, 1982. 